\title{
An Ethical Approach of Block Based Image Encryption Using Chaotic Map
}

\author{
Kamlesh Gupta ${ }^{1}$, Ranu Gupta ${ }^{2}$, Rohit Agrawal ${ }^{3}$ and Saba Khan ${ }^{4}$ \\ ${ }^{1,4}$ Dept. of Computer Science \& Engg., RJIT, Tekanpur. \\ Kamlesh_rjitbsf@yahoo.co.in,saba.khan07@yahoo.com \\ ${ }^{3}$ Dept. of Computer Science \& Engg., JUET Guna \\ juet.rohit@gmail.com \\ ${ }^{2}$ Dept. of Electronics \& Communication, JUET Guna. \\ ranugupta02@yahoo.com
}

\begin{abstract}
In present era all the multimedia communication is done over open network such as Images, Audio, Videos, Text etc, so the security is also a major concern. In this research we proposed an image encryption algorithm by using chaotic map as it is well known for its Dynamic nature, Randomness and very sensitive towards initial condition. In the proposed algorithm two dimensional chaotic map and the two secrets keys for encryption of image are used in which first we divide the image into four blocks and then each block of the image is encrypted individually in $\boldsymbol{n}$ times, after that the keys are inverted for each block and repeat this process up to $m$ times.

The proposed work has been rigorously examined over the prevalent standard test and has encouragingly succeeded to pass most of them like key sensitivity analysis, statistical analysis, differential analysis, entropy analysis, which make the proposed algorithm good enough for real time secure communication.
\end{abstract}

Keywords: Chaotic map, 2-D Logistic map, NPCR, UACI, map, Standard map, Elliptic Curve Cryptography, Curvelet Transform

\section{Introduction}

At present, the image security becomes a world-wide problem which absorbs a great number of researchers to study the robust and secure image cryptosystems to protect the valuable images from leakage.

The images as on date have become an integral and vital component of any useful data and are widely used in several important applications. like Military Image Database \& Message Communication, Confidential Video Conferencing, Medical Imaging System \& Telemedicine, Natural Disaster or Catastrophe Alarming Systems, Online Image Identification and Authentication, Reflection Seismology, Electronic Surveillance Systems, Document Imaging, Image 'CAPTCHA', Image Registration, Geographic Information System etc [9].

Encryption of images is different from that of textual data, as images are intrinsically bulky and have high correlation among pixels and higher redundancy which is difficult to be handled by the traditional encryption schemes. Hence the DES, AES, IDEA, Blowfish, RC6 and RSA etc. do not suite for modern image transmission requirements [10].

Many researchers have tried to innovate better solutions for secured image transmission. In particular, application of chaos theory in multimedia encryption is one of the important research directions.

The aim of this research is to fix the problem for secured image transmission. 
- Asymmetric cryptography doesn't suite for secured transmission of images because

$>$ The bulky data

$>$ Strong pixel correlation

$>$ High redundancy

Moreover encryption at the source and decryption at the destination lowers the encryption performance.

\section{Literature Review}

\subsection{Chaos-Based Image Encryption Schemes}

Recently, a widely studied example of image encryption is based on chaos theory which is well established, simple but complicated dynamics. The chaos functions are used to describe the nonlinear dynamical systems. Chaos function have several interesting properties, these function are very sensitive to the initial conditions which make its importance in data security method. These functions generate random iterative values, these random iterative values are limited between bounds convergence of the iterative values after any value of iteration can never be seen.

In [7], symmetric encryption scheme based on 2D chaotic map is proposed. A two or higher dimensional discretized chaotic maps is adopted for pixel permutation together with 1D map for diffusion. The superiorities of such kind of chaos-based approaches are mainly relatively large block size and a high encryption rate.

The encryption method called CKBA (Chaotic Key Based Algorithm) was proposed in [6]. The algorithm first generates a time series based on a chaotic map, and then uses it to create a binary sequence as a key. According to the binary sequence so generated, image pixels are rearranged and then XOR or XNOR operated with the selected key. This method is very simple but has obvious defects in security, as pointed out lately in $[14,15]$, this method is very weak to the chosen/known-plain-text attack using only one plain-image, and moreover its security to brute-force attack is also questionable. First, sub-keys are used to encrypt more than one block of plaintext, the key set together with binary sequence can be possibly reconstructed through only one pair of known or chosen plain image and encrypted image. Therefore, it cannot resist the chosen and known plaintext attacks. In addition, its security against brute-force attacks is also overestimated by the author due to the fact that the total key length is not fully utilized in the actual encryption. From such point of view, the secret key should never be discussed in all cases.

Color image encryption based on one-time keys and robust chaotic maps was proposed in [11]. One time key cryptosystem based on two robust chaotic maps is designed. The one time pad is a theoretically unbreakable symmetric encryption cipher and true random number generation is much safer than pseudo-random number generator. The proposed cryptosystem has higher security due to an extremely large key space and proposed algorithm combines good confusion and diffusion properties.

The analysis of nonlinear chaotic algorithm (NCA) map and a no. of attacks were proposed in [4]. The weaknesses of image encryption algorithm based on chaotic map describe on the basis of statistical and plain text attacks. Image encryption scheme based on 3D baker with dynamical compound chaotic sequence cipher generator was proposed in [17]. Divide dynamic block of 3D baker map by using compound chaotic map, and compare with 2D baker map. The 3D baker scheme is 2-3 time faster of 2D baker map [17]. The proposed scheme used in real-time secured image transmission.

The cryptanalysis of an image encryption scheme based on a compound chaotic sequence was proposed in [1]. This work combines the confusion/diffusion in single unit for image encryption. Permutation and diffusion are two separate and iterative stages, and they both require scanning the image in order to obtain the pixel values in fastest way. 
Here use the spatio-temporal chaos will be used for fastest image encryption because the period of this chaos is found much longer than that of temporal chaotic maps so that the periodicity problem is practically avoided. The algorithm extracts the pseudo-random numbers directly from the each iteration of the spatial chaos and masks the image data from the first one to the last one, then from the last one to the first one.

The two-dimensional map is proposed in [2]. It is a process of stretch-and-fold. Firstly a square image is divide two parts according diagonal. Secondly each part of image is stretch respectively and joins a line. Lastly the line is fold over to a new square image of same size. The map divides into the left map and the right map. The process shuffles the positions of image pixels. Comparing the prevalent two-dimensional map, cat map and baker map, the new map has simple formulation, big keys space which is fit for image encryption but take larger encryption time. An image encryption scheme based on the map is developed. The numbers of the left map and the right map are used as the key. The de-ciphering process is opposite using the same keys. The results of simulation show that it can be used in real-time image encryption applications.

The efficient chaos based feedback stream cipher for image cryptosystems was proposed in [5]. The proposed stream cipher is based on the use of a chaotic logistic map and an external secret key of 256-bit. The initial conditions for the chaotic logistic map are derived using the external secret key by providing weight-age to its bits corresponding to their position in the key. These proposed features are verified to provide high security level. A complete specification for the proposed ECBFSC is given. Several test images are used for inspecting the validity of the proposed ECBFSC.

According to [12], firstly, chaotic 3D permutation is meaningless if homogenous plain images with identical pixel values are encrypted. In this case, security of the scheme relies merely on a simple diffusion process. Moreover, if a pixel value in the plain images is 0 , then the underlying diffusion operation is also useless. As a result, a key recovery attacks is proposed in such a way that recovers the initial condition of logistic maps according to the gray code. Apparently, the encryption of homogenous plain image is an arbitrarily insufficient issue. However, in [3], this leads to the problem that the scheme is eventually broken with chosen plaintext attacks discussed.

Guan et al. employed the 2D chaotic cat map [19] while Lian et al. employed the 2D standard map [13] for their cryptographic implementation. Fridrich's framework adopts 2D permutation together with simple diffusion process. In 2004, some of mostly used 2D chaotic maps have also been spatially extended to higher dimensional version such as 3D cat map [3], baker map [18] and standard map [13].

The work [8], proposes a new image encryption algorithm using a large pseudorandom permutation which is combinatorial generated from small permutation matrices based on chaotic maps. The random-like nature of chaos is effectively spread into encrypted images by using the permutation matrix. The proposed encryption scheme provides comparable security with that of the conventional image encryption schemes based on baker map or logistic map.

The paper [16], performs a quantitative cryptanalysis on the performance of these kind of ciphers against plaintext attacks.

\section{Proposed Block based Image Encryption}

The Proposed scheme shows efficient image encryption algorithm using 2D chaotic map. The explanation is presenting below in three parts. First we explain how security key is used for finding the initial condition, Secondly the Algorithm and last the procedure used in algorithm for confusion and diffusion. 


\subsection{Methodology}

In this work, we are presenting a new method for image encryption based on 2D logistic map with complicate basin structure and attractor and having more complex chaotic behaviour than one dimensional chaotic map.

The 2D Logistic standard equation

$$
\begin{aligned}
X_{i+1} & =r\left(3 Y_{i}+1\right) X_{i}\left(1-X_{i}\right) \ldots \\
Y_{i+1} & =r\left(3 X_{i+1}+1\right) Y_{i}\left(1-Y_{i}\right) \ldots
\end{aligned}
$$

Where $\left(X_{i}, Y_{i}\right)$ is the pair-wise point at the $\mathrm{i}^{\text {th }}$ iteration and $r$ is the system parameter.

The equation provides random numbers based on the value of $r$. When $r$ lies between (1, 1.11), the attractive focus becomes repulsive and oscillations appears.

- When $r$ lies between $(1.11,1.19)$, it act as a cyclic chaotic behaviours.

- When $r>1.19$, the system becomes unstable

The map act as a pseudo random number generator when at $i=0,\left(r, X_{0}, Y_{0}\right)$ are both known.

In this work a block based symmetric encryption method is used i.e key is same for both the cases either in Encryption phase or in Decryption phase. For diffusing the correlation among pixels first image is divided into blocks then apply the Algorithm over it, so it will shuffle the pixels of each black to make good cryptosystem.

\subsection{Key Convenience}

The key used in this approach is of 256 bit. It's depend on us weather we want to fix the keys or generate the keys randomly at each encryption process. For decryption the key will be same as in encryption process.

One of the most important factors about the key is its length, as the key is the part of the algorithm which is shared between Sender and Receivers. The more the length of the key more it will provide the security to the algorithm. Since the key length in our algorithm is of 256 bit which makes the algorithm better and harder to cryptanalysis.

The key used here is divided into the five parts i.e.

$$
K\left(X_{0}, Y_{0} r, T, A\right)
$$

Where $X_{0}, Y_{0}, r$ and $T$ are denoted as a fractional part for double precision float number of 52 bit length, and $A$ stores the eight initial coefficients for generating random keys each of which contain six byte.

$\mathrm{K}(256)$

$X_{0}=(\mathrm{K}, 1 \ldots \ldots .52)$

$Y_{0}=(\mathrm{K}, 53 \ldots . .104)$

$r=(\mathrm{K}, 105 \ldots \ldots 156)$

$T=(\mathrm{K}, 157 \ldots . .208)$

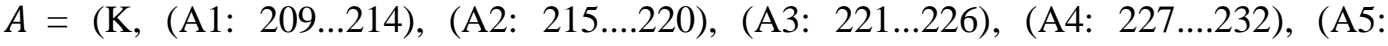

233...238), (A6: 239...244), (A7:245...250),(A8: 251...256))

Both A \& $\mathrm{T}$ are used to generate the $X_{0}$ and $Y_{0}$ among the Procedure i.e $\left(X_{0}{ }^{1}, Y_{0}{ }^{1}, X_{0}{ }^{2}, Y_{0}{ }^{2} \ldots \ldots \ldots \ldots . . . X_{0}{ }^{\mathrm{n}}, Y_{0}{ }^{\mathrm{n}}\right)$

$T X_{0}=\bmod \left(\log (A(\bmod (i-1,6)+1)+i) * X_{0}+T, 1\right)$

$T Y_{0}=\bmod \left(\log (A(\bmod (i-1,6)+1)+i) * Y_{0}+T, 1\right)$ 


\subsection{Algorithm for Block Based Image Encryption}

STEP 1: Take any arbitrary gray scale image $(\mathrm{P})$ having 8 bits / pixel. Resize the image into square dimension like $512 \times 512$.

STEP 2: Divide the image in four blocks of equal size.

$\longrightarrow$\begin{tabular}{|l|l|}
\hline Block 1 & Block2 \\
\hline Block 3 & Block4 \\
\hline
\end{tabular}

STEP 3: Before Encryption, use the properties of XOR operation.

Block $2=$ Block $1 \oplus$ Block 2

Block 3 = Block $2 \oplus$ Block 3

Block $4=$ Block $3 \oplus$ Block 4

STEP 4: Now generate random keys k1 and k2 of size 256 bit length.

STEP 5: Fix the keys for encrypting the blocks i.e

$($ block $1, k 1)($ block $2, k 2)($ block $3 . k 3)($ block $4, k 4)$

\begin{tabular}{|l|l|}
\hline Block 1 (Key1) & Block2 (Key2) \\
\hline Block 3 (Key2) & Block 4 (Key1) \\
\hline
\end{tabular}

STEP 6: Now apply the Procedure (5.4.1) of 2D logistic Diffusion and 2D logistic Confusion over each block using the $2 \mathrm{D}$ logistic equation

$X_{i+1}=r\left(3 Y_{i}+1\right) X_{i}\left(1-X_{i}\right)$

$Y_{i+1}=r\left(3 X_{i+1}+1\right) Y_{i}\left(1-Y_{i}\right)$

This will give the cipher-text image $\mathrm{C}_{1}{ }^{\mathrm{n}}$.

STEP 7: Repeat the step 6 up to $M$ by changing the key which is used previously for block (1, 2, 3 and 4). i.e where key k1 used then used key k2 and if key k2 then k1.

This will return a Cipher-text image $(\mathbf{C})$ with high sensitivity and having correlation near to zero. It will be require that the Cipher image will not contain any relation among the pixels value by the plain image. This can be check by applying certain security checks over the image. 


\subsection{Flow-Chart for Image Encryption}

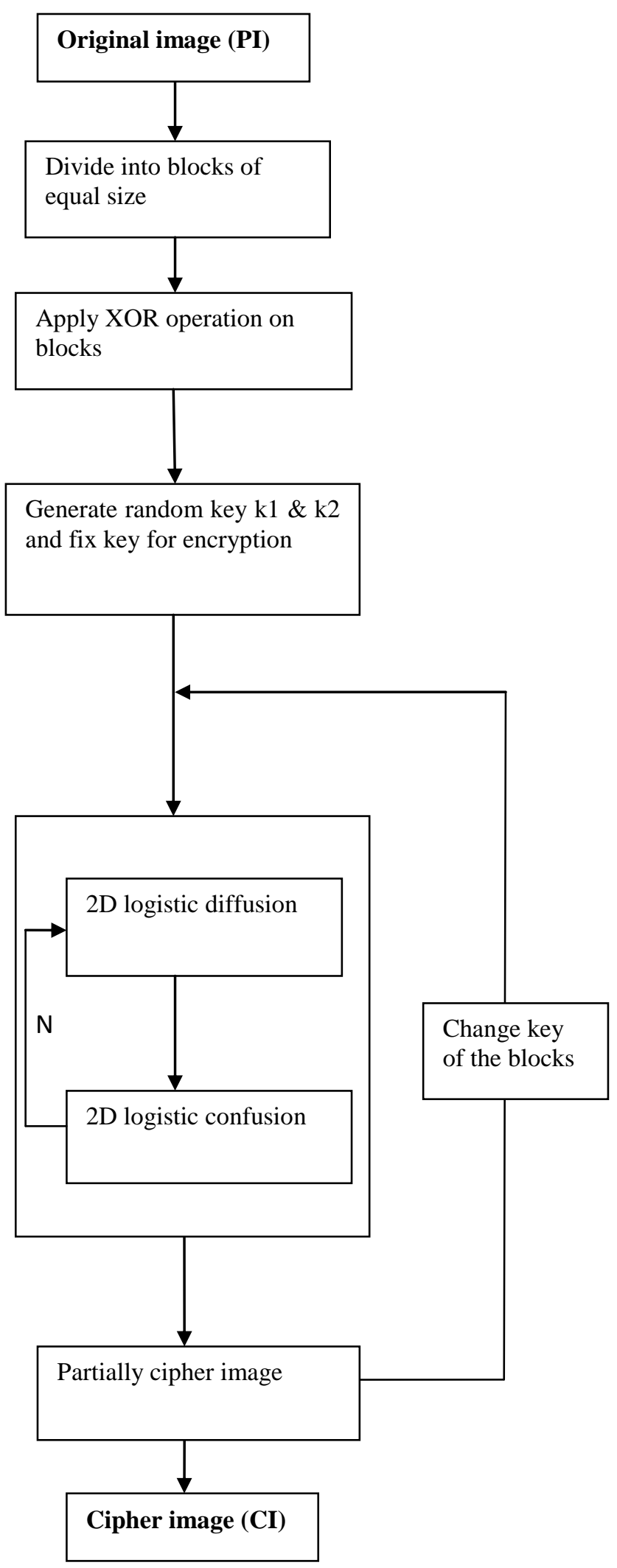




\subsection{Procedure for block Based Image Encryption Using 2-D Logistic Map}

The Procedure is applied over all the four blocks.

Let $\mathrm{P}$ represent the block 1

$p=B 1$ (block1).

Now each $256 \times 256$ block is internally divide as $4 \times 4$ block.

$$
p^{1}=\mathrm{p}=\mathrm{p}_{1} \mathrm{p}_{2} \mathrm{p}_{3} \mathrm{p}_{4} \mathrm{p}_{5} \ldots \ldots \ldots \mathrm{p}_{64}
$$

Now generate a random matrix I of equal size to $\mathrm{P}^{1}$, i.e having 256 rows and 256 colons using logistic map equation having $\mathrm{x}_{0}{ }^{1}$ and $\mathrm{y}_{0}{ }^{1}$ as the initial value and divide that matrix internally in 64 blocks each of size $4 \times 4$.

$$
I^{1}=\mathrm{I}=\mathrm{I}_{1} \mathrm{I}_{2} \mathrm{I}_{3} \mathrm{I}_{4} \mathrm{I} \ldots \ldots \ldots \mathrm{I}_{64}
$$

where each matrix of $I_{1}$ will further changed by the function given below.

$\left(I_{1}, F\right)=\bmod \left(I_{1}(1,1), F\right)$, floor

$\left(\bmod \left(I_{1}(1,2), F\right), \bmod (1,3)^{2}, F\right)$

$\bmod \left(2 * I_{1}(1,4), F\right)$ floor

$\left(\bmod \left(I_{1}(2,1), F\right), \bmod \left(I_{1}(2,2)^{2}, F\right), \bmod (2\right.$

$\left.* I_{1}(2,3), F\right), \bmod \left(I_{1}(2,4), F\right)\left(\bmod \left(I_{1}(3,1)^{2}, F\right)\right.$

$, \bmod \left(2 * I_{1}(3,2), F\right), \bmod \left(I_{1}(3,3), F\right)$, floor $(\bmod$

$\left.\left(I_{1}(3,4), F\right)\right)$

$\bmod \left(2 * I_{1}(4,1), F\right), \bmod \left(I_{1}(4,2), F\right)$, floor $(\bmod$

$\left.\left(I_{1}(4,3), F\right)\right) \bmod \left(I_{1}\right.$

$$
\left.\left.(4,4)^{2}, F\right)\right]
$$

Where $\mathrm{F}$ is the number of allowed intensity scales of the plaintext image. For example, $\mathrm{F}$ $=256$ for a 8-bit grayscale image.

Similarly for,

$I_{2} I_{3} I_{4} I_{5} \ldots \ldots \ldots \ldots \ldots \ldots \ldots \ldots \ldots \ldots I_{64}$

Hence we got new $I^{1}$

Finally, the 2D logistic diffusion is achieved by shifting the each pixel in the plaintext Image with the random integer image $\mathrm{I}^{1}$ over the integer space $[0, F-1]$.i.e. the ciphertext image of $2 \mathrm{D}$ logistic map $\mathrm{C}$ is defined as equation (9)

$$
C^{1}=\left(P^{1}+I^{1}\right) \bmod F
$$

Pass this $C^{1}$ matrix to Confusion procedure to find $C_{1}^{1}$ which is obtain by equation (10)

$$
C_{1}^{1}=C^{1} \oplus X^{1}
$$

Where $X^{1}$ is random image generated by $2 \mathrm{D}$ logistic map using equation $(1 \& 2)$.

Repeat the above Procedure 1.1 up to $N$ iterations as explained below $P^{2}=C_{1}^{1}$

$$
P^{2}=P_{1}^{2} P_{2}^{2} P_{3}^{2} P_{4}^{2} P_{5}^{2} \ldots \ldots \ldots P_{64}^{2} \ldots \ldots
$$

Now generate a random matrix $I^{2}$ of equal size to $P^{2}$. i. e having 256 rows and 256 colons using logistic map equation having $X_{0}^{2} a n d Y_{0}^{2}$ as the initial value and divide that matrix internally in 64 blocks each of size $4 \times 4$.

$$
I^{2}=I_{1}^{2} I_{2}^{2} I_{3}^{2} I_{4}^{2} I_{5}^{2} \ldots \ldots \ldots . I_{64}^{2} \ldots \ldots \ldots \ldots \ldots
$$

Now change the value of $I^{2}$ by using the function use in equation (8)

$I^{2}=I_{1}^{2} I_{2}^{2} I_{3}^{2} I_{4}^{2} I_{5}^{2} \ldots \ldots \ldots I_{64}^{2}$

$$
C^{2}=\left(P^{2}+I^{2}\right) \bmod F \ldots \ldots \ldots
$$

Pass this $\mathrm{C}^{2}$ matrix to Confusion procedure to find $\mathrm{C}_{1}^{2}$ which is obtain by equation (14)

$$
C_{1}^{2}=C^{2} \oplus X^{2}
$$

Where $X^{2}$ is random image generated by $2 \mathrm{D}$ logistic map using equation $(1 \& 2)$

\section{Now at $N^{\text {th }}$ iteration}

$P^{n}=C_{1}^{n-1}$

$$
P^{n}=P_{1}^{n} P_{2}^{n} P_{3}^{n} P_{4}^{n} P_{5}^{n} \ldots \ldots \ldots P_{64}^{n} \ldots \ldots \ldots
$$


Now generate a random matrix $I^{n}$ of equal size to $P^{n}$, i. e having 256 rows and 256 colons using logistic map equation having $X_{0}^{n} a n d Y_{0}^{n}$ as the initial value and divide that matrix internally in 64 blocks each of size $4 \times 4$.

$$
I^{n}=I_{1}^{n} I_{2}^{n} I_{3}^{n} I_{4}^{n} I_{5}^{n}
$$

Now change the value of $I^{n}$ by using the function use in equation (8)

$C^{n}=\left(P^{n}+I^{n}\right) \bmod F$

$$
I^{n}=I_{1}^{n} I_{2}^{n} I_{3}^{n} I_{4}^{n} I_{5}^{n} \ldots \ldots \ldots I_{64}^{n}
$$

Pass this $C^{n}$ matrix to Confusion procedure to find $C_{1}^{n}$ which is obtain by equation (18)

$$
C_{1}^{n}=C^{n} \oplus X^{n}
$$

Where $X^{n}$ is random image generated by $2 \mathrm{D}$ logistic map using eq. 18 and Return this $C_{1}^{n}$ image matrix to step 6 of Algorithm 1, which is a partially encrypted image.

In our proposed method we are encrypting the block by using different key which make our algorithm sensitive and difficult to cryptanalysis.

\section{Result and Performance Analysis}

\subsection{Statistical Analysis}

An ideal cryptosystem should be resistive against any statistical attack. To prove the robustness of our algorithm, we have performed statistical analysis by calculating the histograms in the plain image as well as in the cipher image.

4.1.1 Histogram Analysis: To prevent the leakage of information, it is necessary for the cipher image to bear no statistical similarity to the plain image. An image-histogram describes how the image-pixels are distributed by plotting the number of pixels at each intensity level. The histograms present the statistical characteristics of an image. If the histograms of the encrypted image are similar to the random image, the encryption algorithm has good performance. An attacker finds it difficult to extract the pixels statistical nature of the plain image from the cipher image and the algorithm can resist a chosen plain text or known plain text attacks. Histograms reveal the fact that the random numbers generated from the chaotic map are uniformly distributed. We have shown and analyzed the histograms of the encrypted images along with the original image that have widely different contents.

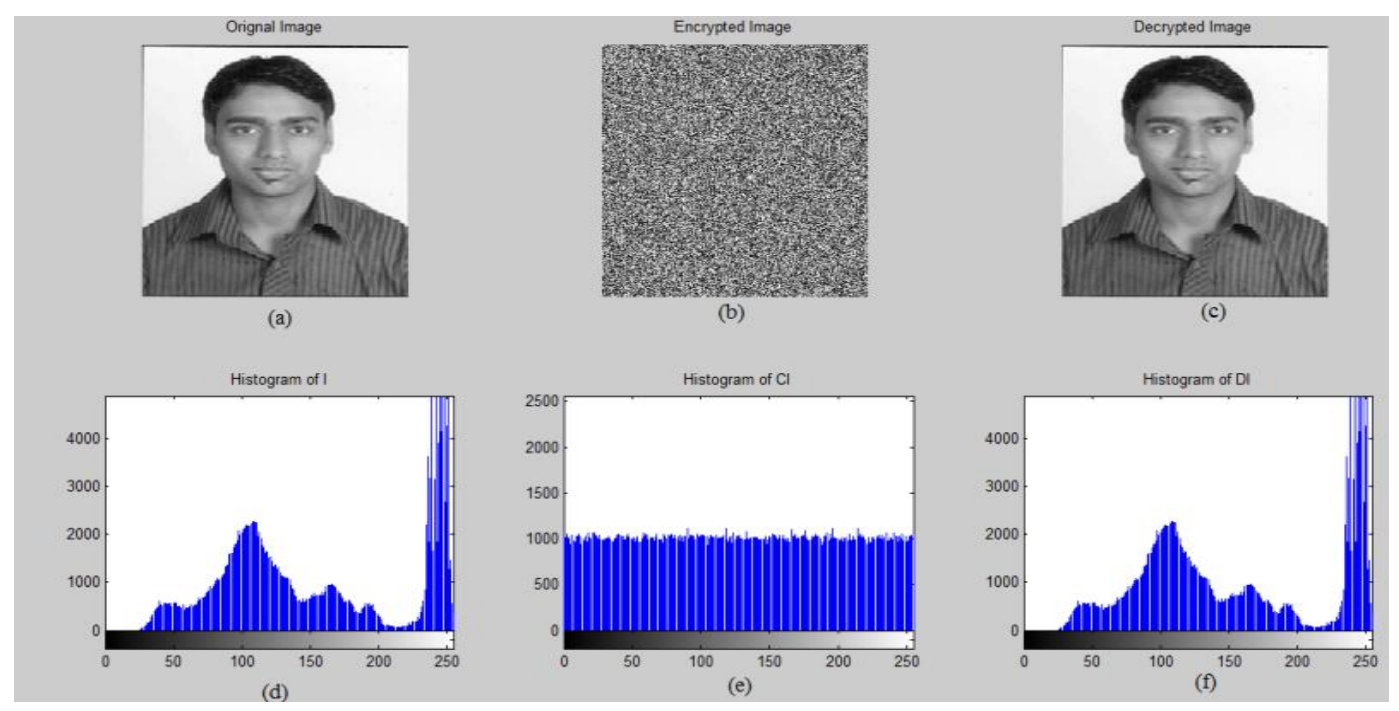

Figure 2. Histogram Analysis result of rohitimage.tif image; (a) Plain-image I \& (d) Histogram of I; (b) Ciphertext image Cl \& (e) Histogram of $\mathrm{Cl}$; and (c) Decrypted image DI \& (f) Histogram of DI 


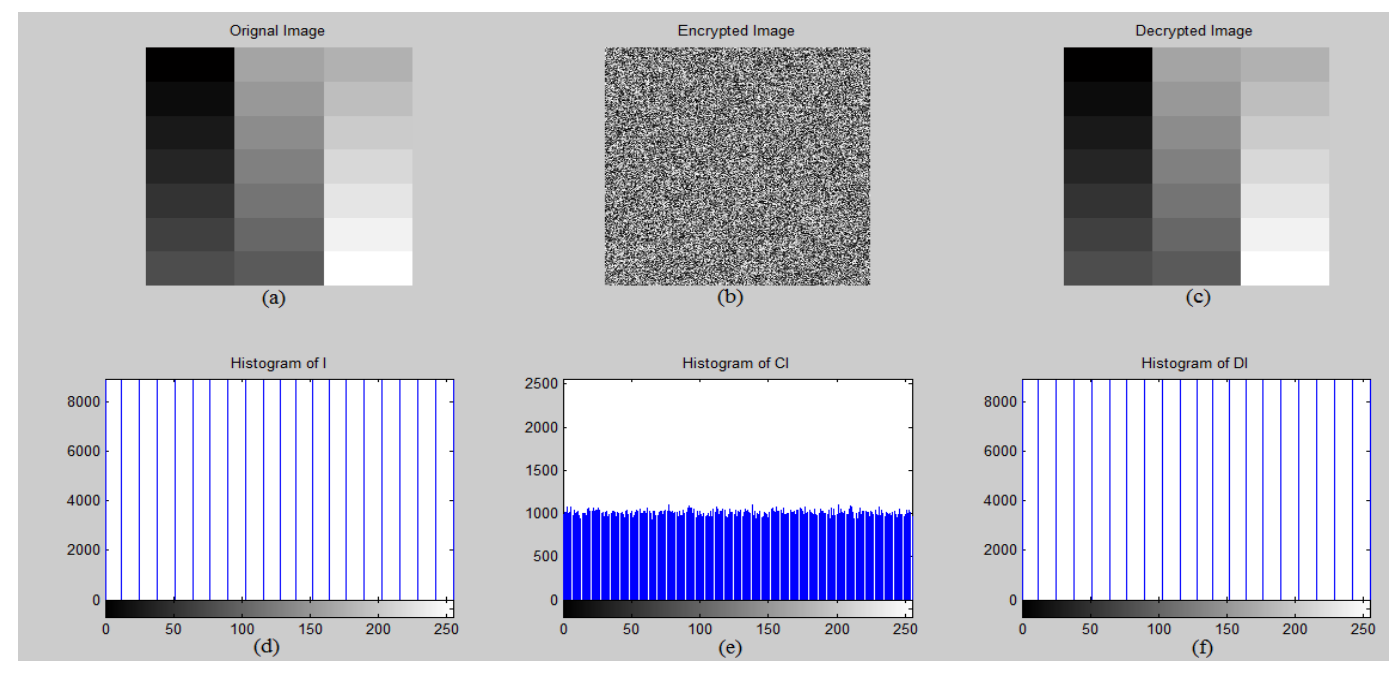

Figure 3. Figure (6.1): Histogram Analysis result of gray21.512.tiff image; (a) Plain-image I \& (d) Histogram of I; (b) Ciphertext image Cl \& (e) Histogram of Cl; and (c) Decrypted image DI \& (f) Histogram of DI

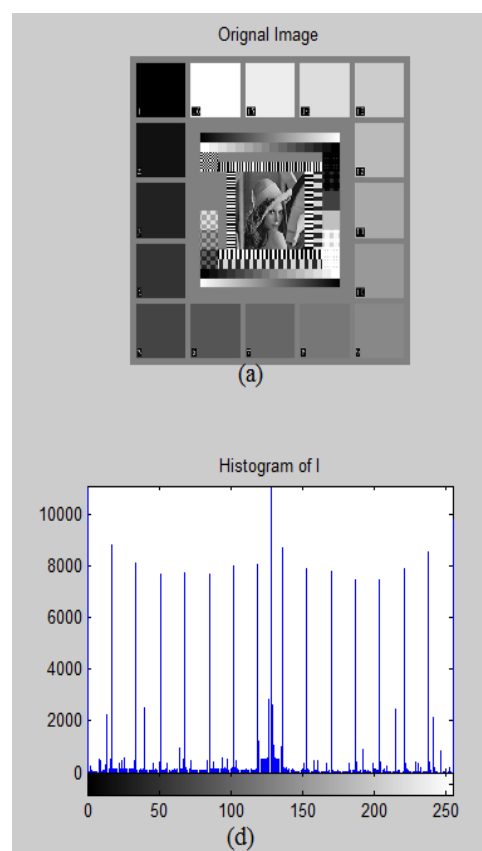

(d)

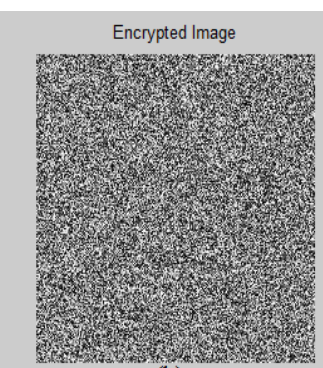

(b)

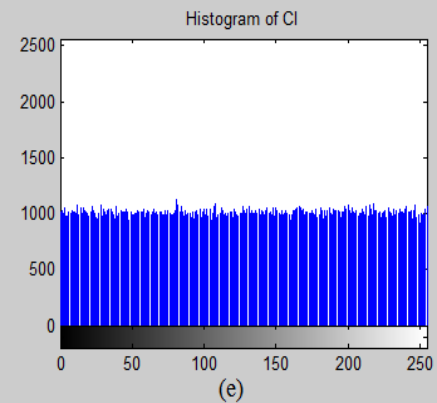

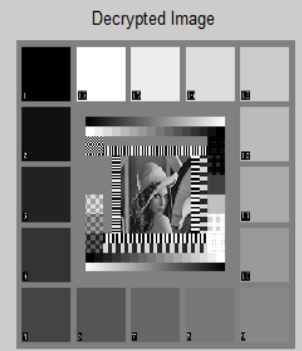

(c)

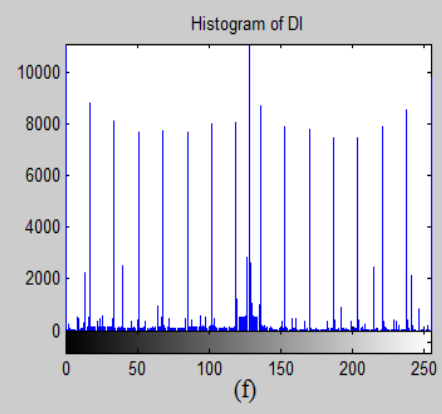

Figure (4): Histogram Analysis result of testpat.1k.tiff image; (a) Plain-image I \& (d) Histogram of I; (b) Ciphertext image Cl \& (e) Histogram of Cl; and (c) Decrypted image DI \& (f) Histogram of DI

4.1.2 Correlation Analysis: The correlation between two vertically adjacent pixels, two horizontally adjacent pixels and two diagonally adjacent pixels in plain.

Image/cipher image are calculated by using the following two formulas:

$$
\begin{gathered}
\operatorname{cov}(x, y)=E(x-E(x))(y-E(y)) \ldots \ldots . \\
r_{x y}=\frac{\operatorname{cov}(x, y)}{\sqrt{D(x)} \sqrt{D(y)}} \cdots \cdots
\end{gathered}
$$


Where $\mathrm{x}$ and $\mathrm{y}$ are the values of two adjacent pixels in the image. In numerical computations, the following discrete formulas were used:

$$
\begin{gathered}
E(x)=\frac{1}{N} \sum_{i=1}^{N} x_{i} \ldots \ldots \ldots \ldots \ldots \ldots \\
D(x)=\frac{1}{N} \sum_{i=1}^{N}\left(x_{i}-E(x)\right)(y-E(y)) \ldots \ldots \\
\operatorname{cov}(x, y)=\frac{1}{N} \sum_{i=1}^{N}\left(x_{i}-E(x)\right)\left(y_{i}-(E(y)) \ldots\right.
\end{gathered}
$$

The correlation coefficient between original and cipher image of horizontal, vertically and diagonally is shown in Table (1). If the correlation of the encrypted image are nearest to zero then it inform good encryption quality.

\section{Table 1. Correlation of Author Image in Horizontal, Vertical and Diagonal Directions}

\begin{tabular}{|l|l|l|}
\hline rohitimage.tif & $\begin{array}{l}\text { Plain- } \\
\text { image }\end{array}$ & $\begin{array}{l}\text { Cipher- } \\
\text { image }\end{array}$ \\
\hline Horizontal & 0.986584 & 0.000605 \\
\hline Vertical & 0.988669 & 0.002165 \\
\hline Diagonal & 0.977623 & 0.003458 \\
\hline
\end{tabular}

\subsection{Key Sensitivity Analysis}

A secure cipher should be sensitive to the encryption key. Such sensitivity is commonly addressed with respect to two aspects:

- Encryption: how different are two Cipher-text image $\mathrm{C}^{1}$ and $\mathrm{C}^{2}$ with respect to the same plaintext image using two encryption keys $\mathrm{K}_{1}$ and $\mathrm{K}_{2}$, which are different only in one bit.

- Decryption: how different are two decrypted image $\mathrm{D}^{1}$ and $\mathrm{D}^{2}$ with respect to the same cipher-text image using two encryption keys $\mathrm{K}_{1}$ and $\mathrm{K}_{2}$, which are different only in one bit.

Figure 5, $6 \& 7$ shows the key sensitivity of the proposed algorithm with respect to Encryption and decryptions where $\mathrm{K}_{3}$ and $\mathrm{K}_{4}$ are differentiate from $\mathrm{K}_{1}$ with only one bit. These results clearly show the $2 \mathrm{D}$ logistic map based image cipher is very sensitive to the encryption key for both encryption and decryption. 


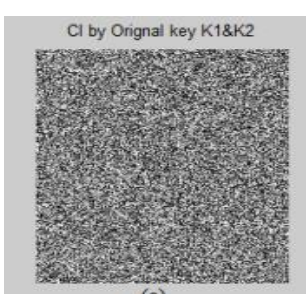

(a)

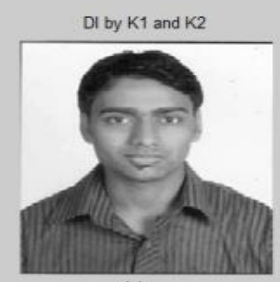

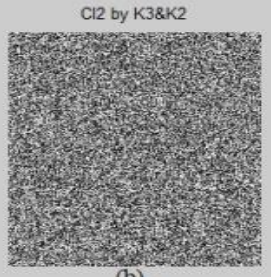

(b)

$\mathrm{D} 12$ by K3\&K2

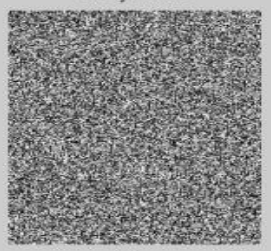

(f)

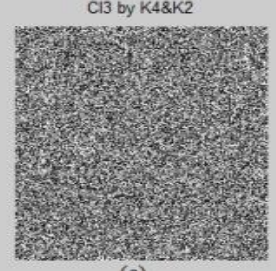

(c)

D13 by K48K2

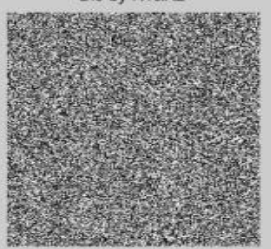

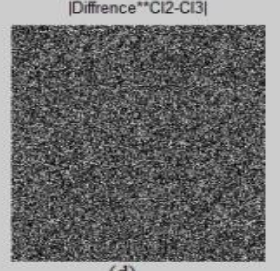

(d)

$|\mathrm{D}| 2-\mathrm{D} 13 \mid$

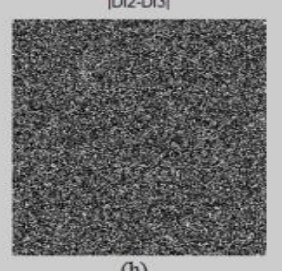

(h)

Figure 5. Key Sensitivity Analysis result of rohitimage.tif image; (a) \& (e) Cipher-image Cl \& Decrypted image DI by original key k1 \& k2; (b) \& (f) Cipher-image Cl2 \& Decrypted image DI2 by modified key k3 \& k2; (c) \& (g) Cipher-image Cl3 \& Decrypted image DI3 by modified key k4 \& k2; (d) Difference in pixels of $\mathrm{Cl} 2 \& \mathrm{Cl} 3(\mathrm{~h})$ Difference in pixels of DI2 \& DI3

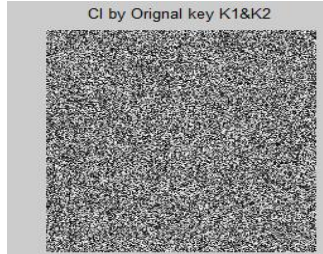

(a)

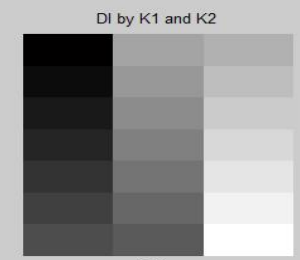

(e)

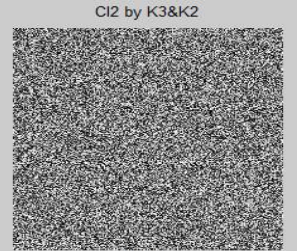

(b)

D12 by K3\&K2

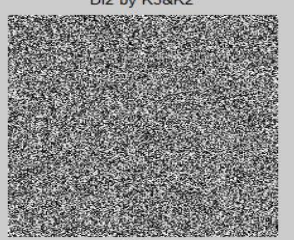

(f)

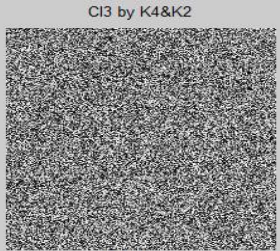

(c)

DI3 by K4\&K2

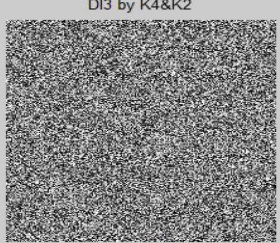

(g)

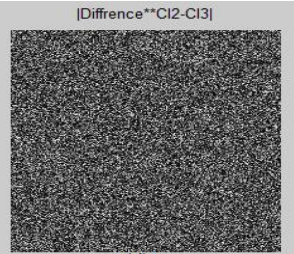

(d)

$|\mathrm{D} 12-\mathrm{D} 13|$

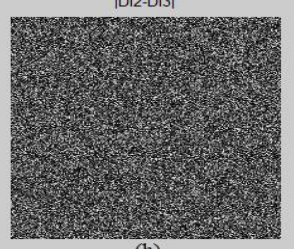

Figure 6. Key Sensitivity Analysis result of gray21.512.tiff image; (a) \& (e) Cipher-image Cl \& Decrypted image DI by original key k1 \& k2; (b) \& (f) Cipher-image $\mathrm{Cl} 2$ \& Decrypted image DI2 by modified key k3 \& k2; (c) \& (g) Cipher-image $\mathrm{Cl} 3$ \& Decrypted image DI3 by modified key k4 \& k2; (d) Difference in pixels of $\mathrm{Cl} 2 \& \mathrm{Cl} 3(\mathrm{~h})$ Difference in pixels of DI2 \& DI3 


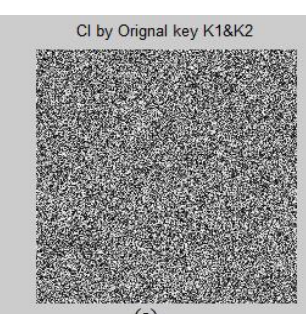

(a)

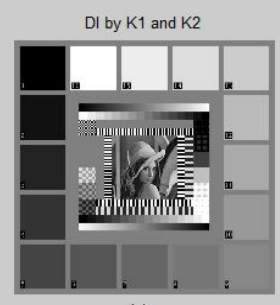

(e)

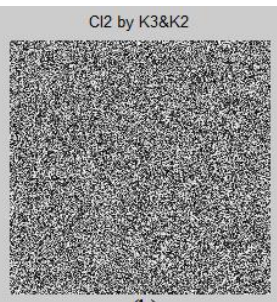

(b)

DI2 by K3\&K2

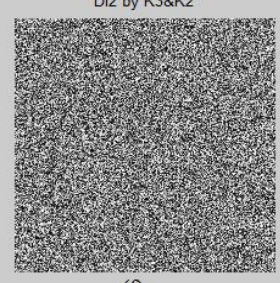

(f)

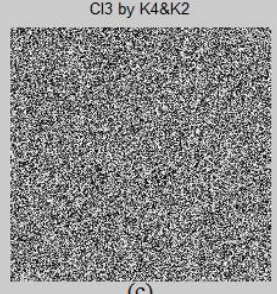

(c)

DI3 by K4\&K2

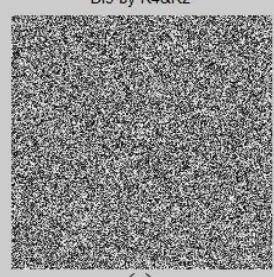

(g)

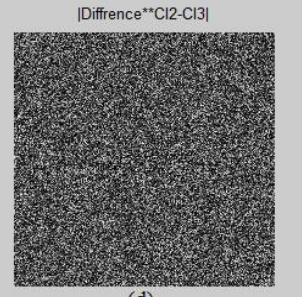

(d)

|D12-D13|

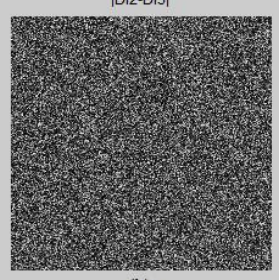

(h)

Figure 7. Key Sensitivity Analysis result of testpat.1k.tiff image; (a) \& (e) Cipher-image Cl \& Decrypted image DI by original key k1 \& k2; (b) \& (f) Cipher-image $\mathrm{Cl} 2$ \& Decrypted image DI2 by modified key k3 \& k2; (c) \& (g) Cipher-image $\mathrm{Cl} 3$ \& Decrypted image DI3 by modified key k4 \& k2; (d) Difference in pixels of $\mathrm{Cl} 2 \& \mathrm{Cl} 3(\mathrm{~h})$ Difference in pixels of DI2 \& DI3

Table 2. Comparisons in which, difference by change in 1 bit in key is shown for Encryption and Decryption

\begin{tabular}{|c|c|c|c|c|c|}
\hline Sr. no & Images & Original key1 & $\begin{array}{l}\text { Randomly modi- } \\
\text { fied } 1 \text { bit of key1 }\end{array}$ & $\begin{array}{l}\text { Pixel dif- } \\
\text { ference in } \\
\text { encryption } \\
(\%)\end{array}$ & $\begin{array}{l}\text { Pixel difference } \\
\text { in decryption } \\
(\%)\end{array}$ \\
\hline 1 & rohitimage.tif & $\begin{array}{l}\text { AEA127A9CC86 } \\
\text { 599D0963DA5E1 } \\
\text { 5579FFE4EE268 } \\
\text { EB0CA6898CB8 } \\
\text { 00225E1E0E7BF } \\
0\end{array}$ & $\begin{array}{l}\text { AEA127A9CC865 } \\
\text { 99D0963DA5E155 } \\
\text { 79FFF4EE268EB0 } \\
\text { CA6898CB800225 } \\
\text { E1E0E7BF0 }\end{array}$ & 99.6109 & 99.6159 \\
\hline 2 & gray21.512.tiff & $\begin{array}{l}\text { 57DE5B9BD51B } \\
\text { 8C5872DE45473 } \\
\text { A3A3AA12300B } \\
\text { 70AD95711C120 } \\
\text { 43EDF09CB4834 } \\
3\end{array}$ & $\begin{array}{l}\text { 57DE5B9BD51B8 } \\
\text { C5872DE45473A3 } \\
\text { A3AA12300BF0A } \\
\text { D95711C12043ED } \\
\text { F09CB48343 }\end{array}$ & 99.6201 & 99.6143 \\
\hline 3 & testpat.1k.tiff & $\begin{array}{l}\text { 939D3E036B354 } \\
\text { 0D4BFC1171FD4 } \\
\text { FFAF9713316802 } \\
\text { 0E843F765D6218 } \\
\text { F52E704104 }\end{array}$ & $\begin{array}{l}\text { 939D3E036B3540 } \\
\text { D4BFC1171FD4F } \\
\text { FAF97133168020 } \\
\text { C843F765D6218F } \\
52 E 704104\end{array}$ & 99.5968 & 99.6063 \\
\hline
\end{tabular}

4.2.3 Differential Analysis Test: In general, a desirable characteristic of an encrypted image is being sensitive to the little changes in a plain image (e.g. modifying only one pixel). Adversary can create a small change in the input image to observe changes in the result. By this method, the meaningful relationship between original image and cipher image can be found. If one little change in the plain-image can cause a significant change in the cipher-image, with respect to diffusion and confusion, then the differential attack actually loses its efficiency and becomes almost useless. 
The NPCR (Noise Pixel Change Ratio) measures the percentage of the number of different pixels to the total number of pixels in these two images. UACI (Unified Average Change Intensity) measures the average intensity of differences between the two images. The higher the values of NPCR and UACI are, the better the encryption.

Consider two cipher-images, $C 1$ and $C 2$, whose corresponding plain-images have only one pixel difference.

The NPCR of these two images is defined in

$$
\mathrm{NPCR}=\frac{\sum_{i, j} D(i, j)}{W \times H} * 100 \%
$$

Where $\mathrm{W}$ and $\mathrm{H}$ are the width and height of the image and $D(i, j)$ is defined as

$D(i, j)=\left\{\begin{array}{l}0, \quad \text { if } C 1(i, j)=C 2(i, j) \\ 1, \quad \text { if } C 1(i, j) \neq C 2(i, j)\end{array}\right.$

Another measure, UACI, is defined by the following formula:

$$
\begin{gathered}
U A C I=\frac{1}{W \times H} \times \sum_{i, j}\left[\frac{C 1(i, j)-c 2(i, j)}{255}\right] \\
* 100 \% \ldots \ldots \ldots \ldots \ldots \ldots
\end{gathered}
$$

Experimentally measured value of NPCR is $99.63 \%$ and UACI is $33 \%$ for Road image. This result indicates that small change in plain image creates significant changes in the ciphered images, so the proposed algorithm is highly resistive against differential attack. Test is applied to check the plain-text sensitivity on the rohitimage.tif, gray21.512.tiff and testpat.1k.tiff in which the pixel difference is shown by changing randomly 1 pixel of Plain image.

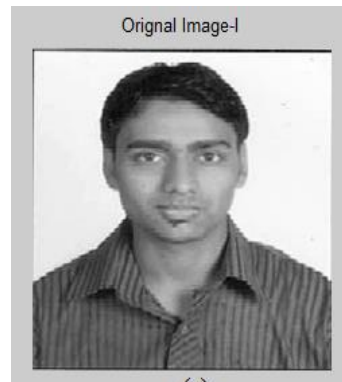

(a)

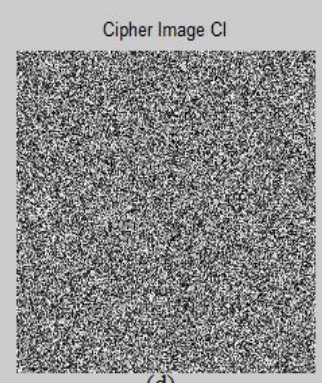

(d)

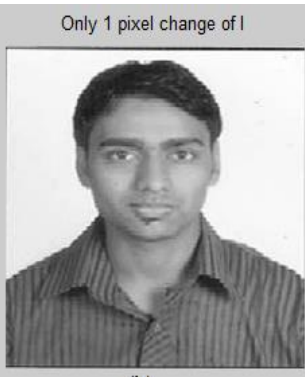

(b)

Cipher Image CJ

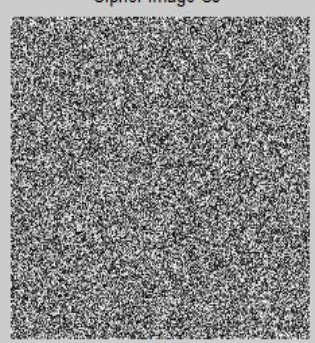

(e)

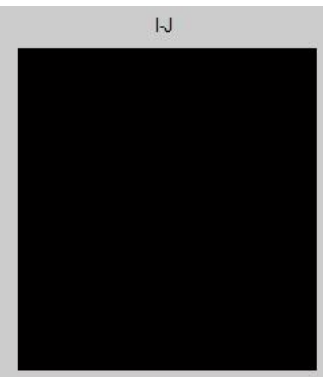

(c)

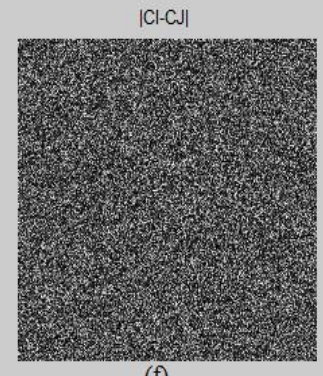

(f)

Figure 8. Plain-text Sensitivity Analysis result of rohitimage.tif image; (a) Plain-image I \& (d) Cipher-image Cl of I; (b) Plain-image I having 1 pixel modified \& (e) Cipher-image CJ of modified I; (c) Difference in pixels between image (a), (b) \& (f) Difference in pixels between $\mathrm{Cl} \& \mathrm{CJ}$ 


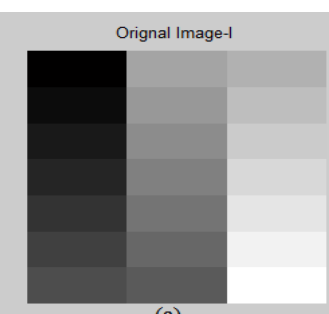

(a)

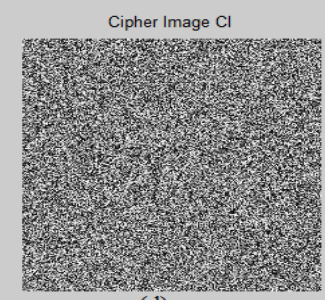

(d)

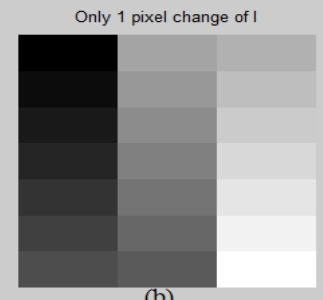

(b)

Cipher Image $C_{J}$

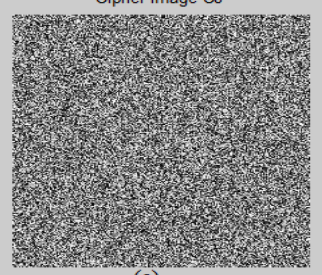

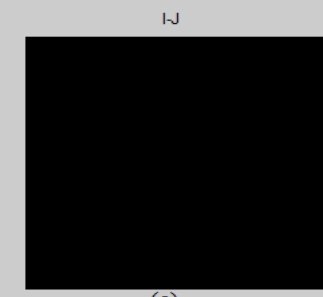

(c)

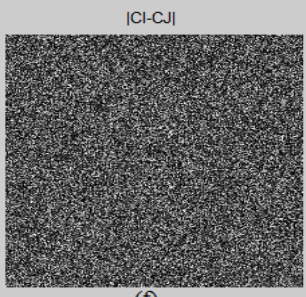

Figure 9. Plain-text Sensitivity Analysis result of gray21.512.tiff image; (a) Plain-image I \& (d) Cipher-image Cl of I; (b) Plain-image I having 1 pixel modified \& (e) Cipher-image CJ of modified I; (c) Difference in pixels between image (a), (b) \& (f) Difference in pixels between $\mathrm{Cl} \& \mathrm{CJ}$

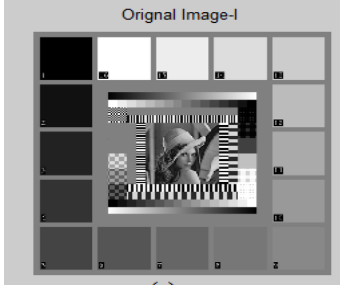

(a)

Cipher Image $\mathrm{Cl}$

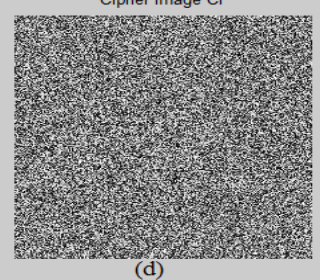

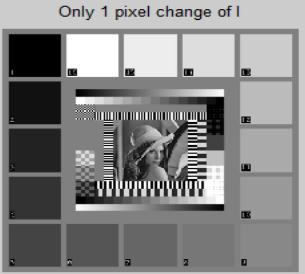

(b)

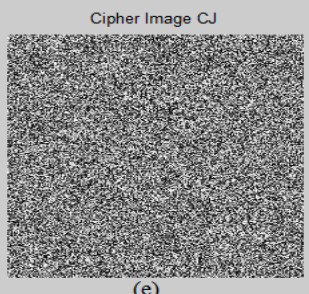

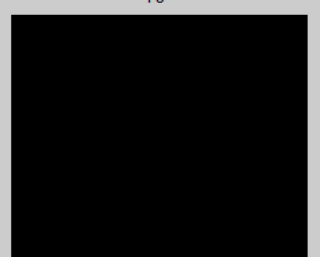

(c)

$|\mathrm{Cl}-\mathrm{C}|$

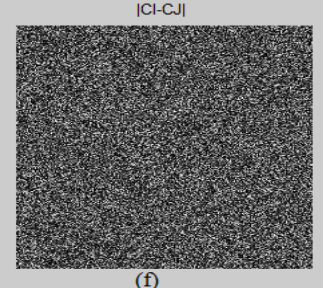

Figure 10. Plain-text Sensitivity Analysis result of testpat.1k.tiff image; (a) Plain-image I \& (d) Cipher-image Cl of I; (b) Plain-image I having 1 pixel modified \& (e) Cipher-image CJ of modified I; (c) Difference in pixels between image (a), (b) and (f) Difference in pixels between $\mathrm{Cl} \& \mathrm{CJ}$

Table 3. NPCR and UACI Test Result

\begin{tabular}{|l|l|l|l|l|}
\hline $\begin{array}{l}\text { S } \\
\text { no. }\end{array}$ & Images & NPCR & UACI & $\begin{array}{l}\text { Test Re- } \\
\text { sult }\end{array}$ \\
\hline 01 & pepper.png & 99.6189117431641 & 33.4613545735677 & pass \\
\hline 02 & rohitimage.tif & 99.6120452880859 & 33.4680445053998 & pass \\
\hline 03 & gray21.512.tiff & $99.6376037597656 \mathrm{e}$ & $33.4843085793888 \mathrm{e}$ & pass \\
\hline 04 & testpat.1k.tiff & 99.6105194091797 & 33.4392562567019 & Pass \\
\hline 05 & fabric.png & 99.6765136718750 & 33.4745100432751 & Pass \\
\hline
\end{tabular}


4.2.4 Information Entropy Analysis: It is well known that the entropy $H(m)$ of a message source $m$ can be measured by

$$
H(m)=\sum_{i=0}^{M-1} p\left(m_{i}\right) \log \frac{1}{p\left(m_{i}\right)}
$$

Where $\mathrm{M}$ is the total number of symbols $m_{i} \in m ; p\left(m_{i}\right)$ represents the probability of occurrence of symbol $m_{i}$ and $\log$ denotes the base 2 logarithm so that the entropy is expressed in bits. For a random source emitting 256 symbols, its entropy is $H(m)=8$ bits. for the different cipher-image, the corresponding entropies should be nearest 7.8 to 8.0. This means that the cipher-images are close to a random source and the proposed algorithm is secure against the entropy attack.

Here we check the entropy by encrypting first by taking the normal image i.e rohitimage.tif whose entropy is 7.0596 then we reduce its entropy up to 2.3851 and 3.7448 by shifting its histogram, as shown in figure $11 \& 12$ and table 4we analysis that our approach will also able to encrypt a low entropy image into higher entropy image having random information content. Hence this will pass the entropy test.

Table 4. Entropy Test Results

\begin{tabular}{|l|l|l|l|l|}
\hline $\begin{array}{l}\text { Sr. } \\
\text { no. }\end{array}$ & Images & $\begin{array}{l}\text { Entropy } \\
\text { Plain Image }\end{array}$ & $\begin{array}{l}\text { Entropy } \\
\text { Cipher Image }\end{array}$ & $\begin{array}{l}\text { Entropy Decrypted } \\
\text { Image }\end{array}$ \\
\hline 01 & rohitimage.tif & 7.0596 & 7.9993 & 7.0596 \\
\hline 02 & Sharper rohitimage.tif & 2.3851 & 7.9994 & 2.3851 \\
\hline 03 & Dark rohitimage.tif & 3.7448 & 7.9993 & 3.7448 \\
\hline
\end{tabular}

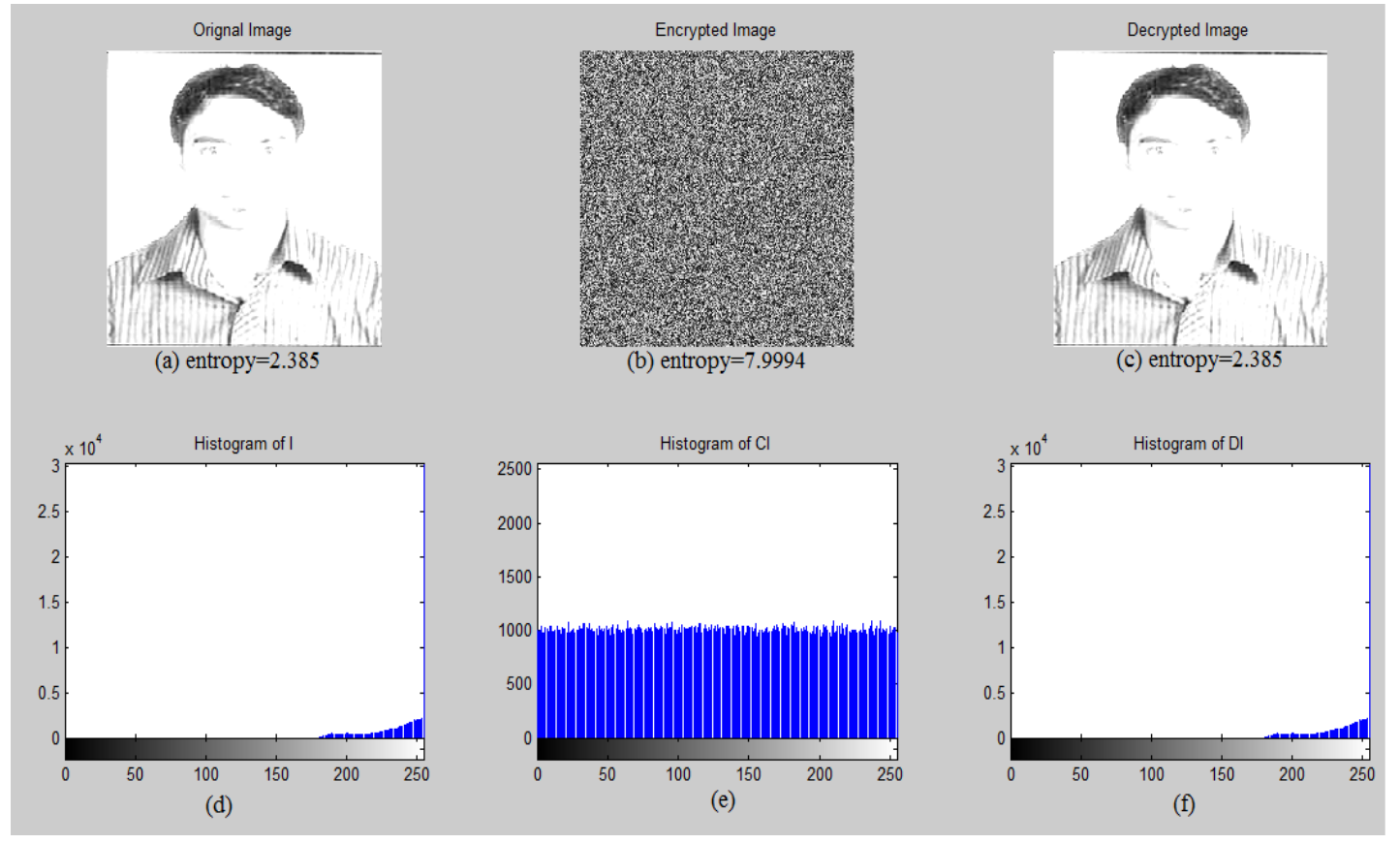

Figure 11. Entropy test result of Sharper rohitimage.tif image; (a) Plainimage I \& (d) Histogram of I; (b) Encrypted image Cl \& (e) Histogram of Cl; and (c) Decrypted image DI \& (f) Histogram of DI 


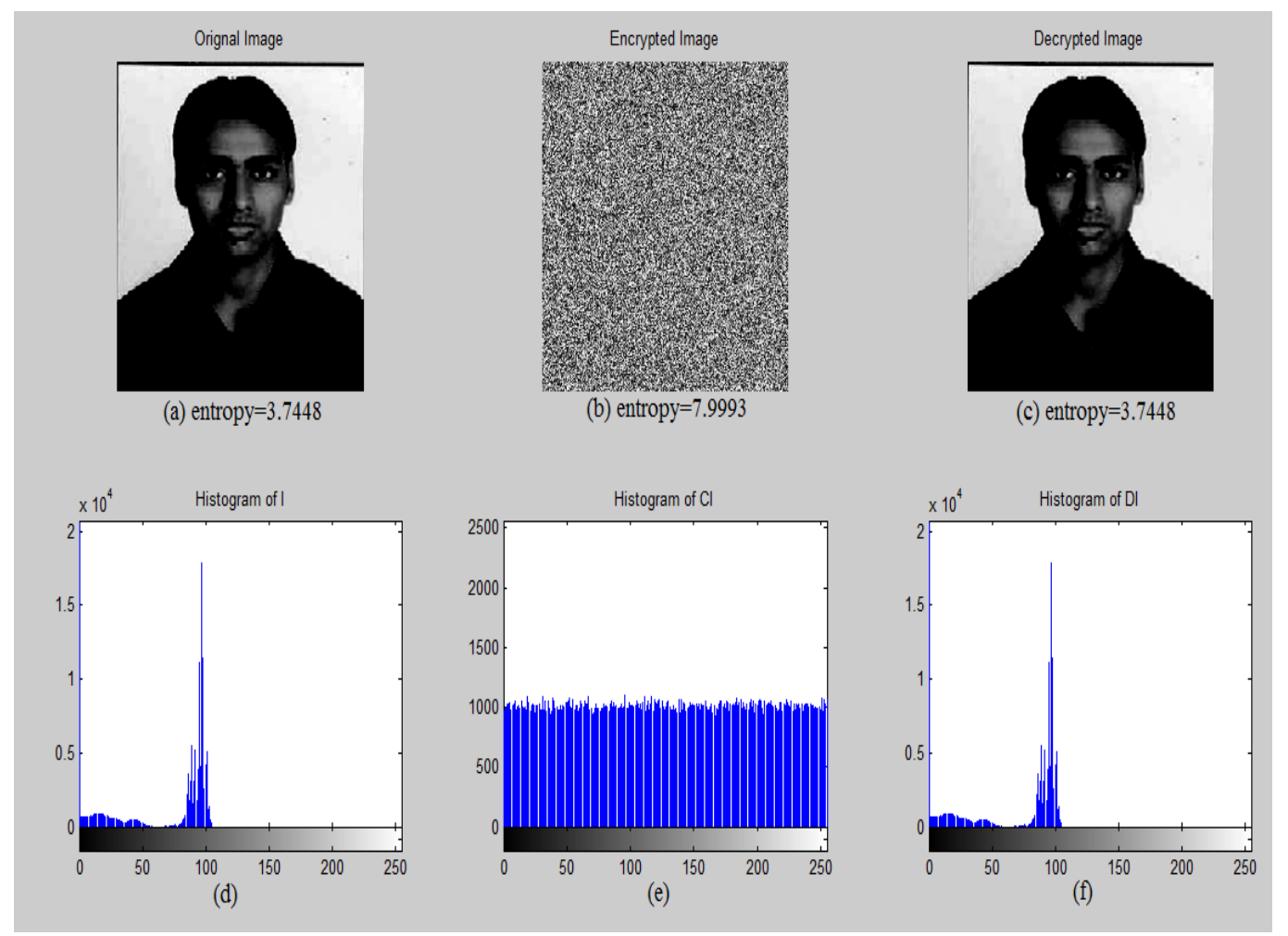

Figure 12. Entropy test result of Dark rohitimage.tif image; (a) Plain-image I \& (d) Histogram of I; (b) Encrypted image Cl \& (e) Histogram of $\mathrm{Cl}$; and (c) Decrypted image DI \& (f) Histogram of DI

\section{Conclusion}

In this research, we proposed an image encryption algorithm using chaos mapping and we showed that this algorithm could hide the original image through simple permutation of the pixels location as well as transformation of the gray scale value through Boolean XOR operation. To make the cipher more robust against any attack, the secret key is exchange after encrypting each blocks of the image. We have carried out statistical analysis, key sensitivity analysis, keyspace analysis and entropy test analysis to demonstrate the security of the new image encryption procedure. Finally, we conclude with the remark that the proposed method is expected to be useful for real time image encryption and transmission applications.

\section{References}

[1] C. Li, S Li, G C,W and A. Halang, "Cryptanalysis of an Image Encryption Scheme Based on a Compound Chaotic Sequence (2009)", Image and Vision Computing, vol. 27, no. 8, pp. 1035-1039, Elsevier. DOI: 10.1016/j.imavis.2008.09.004, (1993).

[2] F. Huang and Y. Feng, "An Image Encryption Approach Based on a New Two-dimensional Map", IEEE 0-7695-2745-0/06, (2006).

[3] G. Chen, Y. Mao and C. K. Chui, "A symmetric image encryption scheme based on 3D chaotic cat maps", Chaos Solitons and Fractals, vol. 21, (2004), pp. 749-761.

[4] G. Alvarez and Shujun Li, "Crypt analyzing a nonlinear chaotic algorithm (NCA) for image encryption", Communication Nonlinear Sci. Numer. Simulation, vol. 14, (2009), pp. 3743-3749.

[5] H. E.-d. H. Ahmed, H. M. Kalash and O. S. Farag Allah, "An Efficient Chaos Based Feedback Stream Cipher (ECBFSC) for Image Encryption and Decryption.

[6] J. C. Yen and J. I. Guo, "A New Chaotic key-based design for image encryption and decryption", Proc. of IEEE International Symposium Circ. System, vol. 4, (2000), pp. 49-52.

[7] J. Fridrich, "Symmetric ciphers based on two-dimensional chaotic maps", Int. J. Bifurcat. Chaos, vol. 8, no. 6 , pp. $1259-1284$. 
[8] J. W. Yoon and H. Kim, "An image encryption scheme with a pseudorandom permutation based on chaotic maps", Communication Nonlinear Sci Numer Simulation, (2010), pp. 1-9.

[9] K. Gupta and S. Silakari "Novel Approach for fast Compressed Hybrid color image Cryptosystem", Advances in Engineering Software, Elsevier, vol. 49, (2012), pp. 29-42.

[10] K. Gupta and S. Silakari, "New Approach for fast color image encryption using chaotic map", Journal of Information Security, vol. 2, no. 2, (2012), pp. 139-150.

[11] H. Liu and X. Wang, "Color image encryption based on one-time keys and robust chaotic maps", journal of Computers and Mathematics with Applications, vol. 59, (2010), pp. 3320-3327.

[12] S. G. Lian, J. Sun and Z. Wang, "Security analysis of a chaos based image encryption algorithm", Physica A, vol. 351, (2005), pp. 645-61.

[13] S. G. Lian, J. Sun and Z. Wang "A Block Cipher Based on a Suitable use of Chaotic Standard Map", Chaos, Solitons and Fractals, vol. 26, no. 1, (2005), pp. 117-129.

[14] S. Li, C. Li, G. Chen, G. Bourbakis and K. T. Lo "A General Cryptanalysis of Permutation-Only Multimedia Encryption Algorithms", Available in IACR's, http://eprint.iacr.org/2004/374., (2004).

[15] S. Li and X. Zheng, "Cryptanalysis of a Chaotic Image Encryption Method", Proc. of IEEE International Symposium Circ. Syst., vol. 2, (2002), pp. 708-711.

[16] S. Li, C. Li, G. Chen, N. G. Bourbakis and K.-T. Lo, "A general quantitative cryptanalysis of permutation-only multimedia ciphers against plaintext attacks", Signal Processing Image Communication, vol. 23, (2008), pp. 212-223.

[17] X. Tong and M. Cui, "Image encryption scheme based on 3D baker with dynamical compound chaotic sequence cipher generator”, Signal Processing, vol. 89, (2009), pp. 480-491.

[18] Y. B. Mao, G. Chen, S. G Lian, "A Novel Fast Image Encryption Scheme Based on 3D Chaotic Baker Map", International journal of Bifurcation and Chaos, vol. 14, no. 10, (2004), pp. 3613-3624.

[19] Z. H Guan, F. J. Huang and W. J. Guan, "Chaos-based Image Encryption Algorithm", Physics Letter A, vol. 346, (2005), pp. 153-157. 
International Journal of Security and Its Applications

Vol.9, No.9 (2015) 